\title{
Young researchers conference of the Centre for Research on Social Interactions
}

Letizia Volpin, University of Neuchâtel, Institute of language sciences and communication* Klara Skogmyr Marian, University of Neuchâtel, Institute of language sciences and communication Fabienne Gfeller, University of Neuchâtel, Institute of psychology and education Sylvia Gonzalez, University of Neuchâtel, Institute of language sciences and communication Adrian Bangerter, University of Neuchâtel, Institute of work and organizational psychology

${ }^{*}$ Corresponding author: letizia.volpin@unine.ch

\section{Introduction}

Social interaction plays an important role in the daily life of every human being. Whether it is within the private sphere of the home or in public spaces, communication constitutes an essential part of human life and its development. Thus, studying and modelling this phenomenon is of crucial importance, not only in the interests of individuals, but also for the proper functioning of our institutions. In the past few decades, there has been a growth in the research on social interaction, with a vast number of empirical studies from such diverse disciplines as linguistics, psychology and biology. These studies rely on different epistemological and theoretical frameworks and use a large range of qualitative and quantitative methodological tools. Faced with this heterogeneity, it becomes important to bring together the main approaches used to uncover the processes underlying this multidimensional phenomenon, in order to establish a common ground and facilitate interdisciplinary collaborations.

The Centre for Research on Social Interactions (CRSI) of the University of Neuchâtel emerged as an interdisciplinary (and inter-faculty) collaboration built around the study of social interaction ${ }^{1}$. The CRSI doctoral program, in turn, was

1 Links to the CRSI websites:

https://www.unine.ch/centres-of-excel lence/home/interactions_sociales.html; https://www.unine.ch/interactionssociales. created to provide $\mathrm{PhD}$ students with theoretical and methodological support in their research endeavours. Within this framework, the Young Researchers Conference of the Centre for Research on Social Interaction (the CRSI-YR Conference) was organised on February 16-17, 2017, as a concrete effort to offer young researchers working on social interaction an opportunity to present their work and to learn from experienced scholars in an environment that fosters dialogue across academic disciplines. The conference brought together keynote and invited speakers as well as student presenters representing a wide range of topics and approaches related to social interaction. In total, 53 participants from different universities in Switzerland and abroad attended the conference. Among them, $28 \mathrm{PhD}$ students and other young researchers presented their ongoing work. There were four keynote speakers: Prof. Jean-Marc Dewaele (Birkbeck College, University of London, UK), Prof. Sara Greco (University of Lugano, Switzerland), Prof. Michèle Grossen (University of Lausanne, Switzerland), and Prof. Klaus Zuberbühler (University of Neuchâtel, Switzerland), as well as five invited speakers: Prof. Beatrice Ligorio (University of Bari, Italy), Dr. Eric Mayor (University of Neuchâtel, Switzerland), Prof. Gaëlle Molinari (University of Geneva, Switzerland), Dr. Cécile Petitjean (University of Neuchâtel, Switzerland), Dr. Sonia Szramek-Karcz (University of Silesia in Katowice, Poland).

In this review article, we provide an overview of the main themes and issues 
addressed at the conference. To do so, we summarise the 35 oral and poster presentations (a list of presenters is provided as supplementary material for this review at www.scoms.ch) under five headings regrouping the main topics that emerged from the variety of presentations. Because of the intrinsically interdisciplinary nature of social interaction studies, some of the presentations will be addressed under more than one theme. The five themes are of course not a definite and universal classification, but an attempt to organize the diversity of issues addressed during the conference.

\section{Socio-cognitive mechanisms and affective dimensions of communication}

A part of the conference was devoted to research dealing with the mental mechanisms and communicational strategies underlying and manifested through social interaction. These studies represent various academic disciplines ranging from life sciences to humanities. The empirical work presented here addressed issues on the evolutionary origins as well as on the cognitive, motivational and communicational processes involved in social interaction.

In the opening talk of the conference, Zuberbühler addressed the question of the evolutionary origins of language through a review of empirical work on primate communication. This presentation highlighted the fact that goal-directed intentional communication, like signalling a danger to someone else, exists within both human and great apes interactions, while shared intentionality (to take into account each other's mental states) seems to be exclusively a human capacity. Monier and Avdiji also focused on this ability (sometimes referred to as "theory of mind," "shared knowledge" or "mindreading”, by proposing a cooperative perceptual categorisation task produced in a situation of verbal and non-verbal interaction (Monier), and by testing a tool based on pragmatic the- ories of alignment (considering oral communication as a joint activity, Avdiji).

As a second point, a number of studies represent the new surge of interest in social interaction research concerning the influence of affective dimensions on different aspects of communication. Starting with the main argument that emotions play a central part in our lives, Dewaele presented an overview of recent research on emotion and multilingualism from various methodological and theoretical backgrounds. One main finding of such research relates to the influence of affective states on communication in a foreign language. Also about stress effects in communication, Mayor questioned the effect of gender traits (masculinity and femininity) on the appraisal of different types of stressful events by measuring physiological stress manifestations (heart rate) in a verbal and cognitive task. Gonzalez used the same psychosocial stress induction paradigm with a cooperative storytelling task in order to assess its effect on a speaker's referential productions that are theoretically linked with adjustment ability in discourse. In the same current of studies, evidence from research on speech pathology shows that pervasive emotions such as social anxiety may be one of the main causal factors of some communication disorders such as selective mutism. With the objective to refine diagnostic criteria of this syndrome, Opryszek presented a case study reviewing new criteria of what her research group calls "emotional speech block.” Finally, Petitjean's conversation analytic research about laughter in interaction illustrated a different perspective on issues that are otherwise often considered in terms of speakers' emotional and affective states. Focusing purely on the surface manifestations of this phenomenon in different communicational settings, Petitjean highlighted how laughter can function as a powerful resource to manage multiple aspects of social interaction in both institutional contexts (the classroom, at work) and texted-based conversations (WhatsApp messages).

A last group of presentations on this theme addressed the effect of different 
affective dimensions on collaborative processes and learning. Based on the assumption that emotion awareness encourages co-workers to engage in various processes leading to growing outcomes in work environments, Molinari presented on the role of emotion perception in a computer-mediated collaboration task. This work provides basis for the creation of new tools that improve collaborative processes of people working together through computers. Adopting a developmental approach, Dukes tried to answer how culture can be transmitted and perpetuated over generations by testing through different experiments the assumption that infants use socio-cognitive and affective processes (e.g. appraisal processes) to categorise objects. Finally, Sebiane proposed an experiment testing the impact of music on the learning of a set of simple non-functional behaviours (gestures without a function) and showed that emotion can have an influence on memory and learning in the elderly.

\section{Learning and social interaction}

Numerous presenters work on issues related to learning, trying to understand e.g. the role of social interaction in learning or conversely how competences needed in social interaction are learnt. Among these, several researchers presented studies taking place in formal educational settings. Grossen, for example, presented an analysis of classroom interaction with the aim of broadening our understanding of the notion of interaction from a dialogical perspective considering the interaction as a whole as the basic unit of analysis rather than focusing on an individual's activity. Machado and Cesar focused more on learning processes, by underlining the role of the first language in mathematical reasoning, and therefore in school success/ failure, among bilingual children, while Kronfli analysed communication in a debrief situation in simulation-based education for health professionals. Ligorio also presented a project taking place in a formal educational context, that is, the universi- ty. With a more interventionist approach, which not only aims to understand but also to propose and implement new tools, she drew on scientific knowledge about social interaction and learning in order to create and improve an interactive course with the help of new technologies. This interventional dimension was also important in the project presented by Macedo, who analysed a course that was created as part of a research project in order to study argumentation at the university. Still concerning higher education, Shafei analysed some more macro-level interactional dynamics in educational policies in Egypt.

But learning does not take place solely in formal educational settings, as illustrated by Skogmyr Marian's work, which analysed some aspects of second language learning taking place in the host family of an au-pair. Finally, Molinari presented a study on the role of communication of emotions in computer-mediated learning. Doing this she brought together three themes that were recurrent during the conference: learning, emotion (see also Section 2) and new technologies (see also Section 4).

To sum up this section, the abovementioned presentations highlight the important role of learning-related inquiries in communication research, while also illustrating some of the many ways that learning processes can be studied in such research. By focusing on different social contexts and drawing on a variety of methodological tools, seen together, these studies help us gain a better understanding of the role of communication in learning processes, in particular, how knowledge of social interaction can be used to foster learning, and how we learn competences and skills mobilised in social interaction. Finally, this research also shows how we may study not only the cognitive and social processes related to learning, but also the role of emotions and appraisal processes in learning situations. 


\section{Social practices in naturalistic settings}

Many of the presented studies reconvene in their interest in the social and interactional practices of participants partaking in everyday activities. That is, instead of analysing elicited interactional data in experimental settings or focusing on the mental, cognitive or affective aspects of social interaction like many of the abovementioned studies, these studies were based on naturally occurring or only marginally controlled interaction, and focused primarily on the observable surface manifestations of communication. Several of the presentations belonged to the field of argumentation studies, which aims to document people's discursive argumentative practices. Greco's lecture provided a useful introduction to the field, by presenting empirical examples of this type of research from various social settings including dispute mediations. Schär explored argumentation practices in discussions between young children and their parents, while Macedo analysed how an educational setting aiming to provoke argumentative interaction may enhance students' cognitive development. The connection to argumentation practices can, to some extent, be seen in Piotrowska's study of political radio interviews - a context in which rather fierce adversarial sequences between journalists and interviewees often occur, while Skogmyr Marian's presentation on directive sequences shed some light on L2 speaker's practices for solving similarly precarious situations in family interaction.

In addition to the abovementioned studies that take an interest in a similar object of study (i.e., argumentation practices and related issues), other presentations have in common their conversation analytic approach, which seeks to uncover the systematic organization of people's intersubjectivity efforts, or their interest in social practices in a specific setting or medium of interaction. Chernyshova's paper on collaborative explicitation sequences in ordinary French conversation as well as Bengsch's study of interactional sequenc- es between participants at international hotels' front desks showed how sequential analysis of the unfolding interaction help our understanding of the fine-grained coordination that participants continuously and routinely do to attain intersubjectivity in different social settings. Steger's and Hochuli's studies further addressed the multimodal aspects of such social conduct, by focusing on patterns of lingering (Steger) and rhythms of movements (Hochuli) in public spaces. These studies highlight the embodied nature of social coordination, which takes place both at the micro-level through e.g. gestures and facial expressions, and in the way we organise our patterns of walking, sitting, etc. at the market, in squares, and in other public places.

Petitjean's talk on laughter as an interactional phenomenon exemplified how sequential micro-level analysis can be used to reveal patterns of everyday social conduct (such as laughter) both in various types of face-to-face interaction (educational settings, speech therapy sessions, etc.) and in technology-mediated written communication (WhatsApp conversations). The timely issue of technologymediated interaction was also addressed by other presentations, including Khonineva's study of problems of understanding in human and digital voice assistant interaction, and Fan's presentation on the role of digitally retouched selfies in internet communication. While much of prior research on people's use of technological devices has been based on self-report data and quantitative measures, the presented studies (particularly Petitjean's and Khonineva's studies) demonstrated the value of a qualitative, social-interactional analytic approach that focuses on people's micro-level conduct when they communicate with each other through a text-based medium and with digital voice assistants.

In sum, among the studies discussed above, some focused on uncovering practices for accomplishing specific activities (e.g. argumentation), others on interactional patterns of particular settings (e.g. the hotel front desk), and other yet on people's practices while communicating through a specific medium (e.g. via Whats- 
App). What they all have in common is their interest in attempting to understand and describe different aspects of basic human sociality in naturalistic settings.

\section{Social interaction in multilingual contexts}

Today, the majority of the global population speaks more than one language. Individuals of all ages and all social backgrounds communicate increasingly cross-culturally, in languages other than their first language, and by mixing different languages in the same conversation.

In one way or the other, feelings are omnipresent in our daily life - including when we communicate with others. Therefore, emotions may be seen as part of the construct of interactional competences, as Dewaele argued in his keynote speech on multilingualism and emotions. The use of a foreign language to communicate with others is influenced by our feelings and emotions, and Dewaele demonstrated different quantitative and qualitative approaches to investigate this issue. The expression of emotions through language is also linked to the process of acculturation of multilingual speakers. Tilston addressed the issue of culture in presenting a comparison between the emotions expressed by individuals from two different types of cultures: a collectivist one and an individualist one. Her results help us better understand the role of culture and emotions in communication with strangers. In the case of Polish broker teenagers living in the UK, Zytowicz's study indicated that the act of translating to a third person something written in a foreign language into his/her language (language brokering) is an experience that triggers both positive and negative emotions.

Another factor that influences the communication among multilingual individuals in various ways is the degree of proficiency in a language. Szramek-Karcz explored the topic of intentional and non-native bilingualism in communication among parents and children and discussed the effects of these practices on for- eign language learning. In addition, three more researchers presented their studies about children in a multilingual context. On the one hand, Machado and César focused on the role of the first language and culture for the way of reasoning around and solving mathematical problems at school. They focused on an instrument to assess these skills, taking into account the linguistic and cultural background of students. On the other hand, Paze presented her project about the linguistic assessment of bilingual children with speech and language impairments through narration tasks and with the scaffolding of adults.

These presentations shed some light on the complex topic of bi- and multilingual communication between individuals as well as on various factors involved in this phenomenon, including the role and expression of emotions in second language communication.

\section{Methodological and epistemological issues}

Many papers described their methodology in detail. This brought us through many different methods during these two days, ranging from experimental settings to more ethnographical research. Nevertheless, some researchers addressed methodological and even epistemological issues more explicitly, discussing the question of how we can study social interaction, what kind of data we produce and how we may analyse such data. Through the presentation of a dialogical approach (see Section 3 for a definition) to social interaction, Grossen, notably, raised the issue of unit of analysis, but also of what can be understood and studied under the words "social interaction." Ventura and César also presented a critical point of view on research methods, underlining the link between methods and results. They especially highlighted how each method gives a voice to some groups participating in the research and not to others, which raises issues of power and which will shape the outcomes of the research. Doing this, they questioned our (sometimes implicit) un- 
derstanding of the role of the participants. To what extent do they participate in the research process itself?

Several presentations also provided through their research designs some interesting starting points to nourish debates about different epistemological and methodological approaches. Brandenberger and Hottiger, for example, proposed the use of video and eye-tracking technology in social interaction research, highlighting the challenge of a successful data collection, the technical questions involved in it but also the necessary choices to make in this step of the research process.

Also from a methodological point of view, some of the research presented at the conference focused more on the position of researchers in scientific work. To what extent do researchers' activities constitute an intervention? More broadly, these presentations also invited us to reflect upon the role of the researcher in society by thinking about the practical applications of the research in different domains of life. Projects like Avdiji's, which studies the implementation of a team coordination tool with reference to a design approach, highlight the issue of the relation between theory and "the field." From a clinical perspective, Pilch contributed to this debate with her case study aimed at proposing a new evaluation tool for clinical practice with children having selective mutism.

Finally, the presence of researchers from linguistics, such as Perusset, who presented a semiotic categorisation (e.g. "agent of the action") making possible the analysis of practices, allowed us to reflect on the process of conceptualisation. These are only a few examples of the diversity of methodological and epistemological issues raised throughout the conference; nevertheless, these examples were discussed here because they are either particularly explicit or particularly illustrative for these questions.

\section{Closing remarks}

This review attempted to capture a twoday program of oral presentations, panel discussions and interactive dialogue at the CRSI-YR, and to bring attention to both the heterogeneous and complementary nature of the many different topics investigated and the theoretical and methodological approaches used in the study of communication. The five headings representing the main themes of the conference (socio-cognitive and affective dimensions of interaction, learning and social interaction, social practices in naturalistic settings, interactions in multilingual contexts and methodological and epistemological issues) helped us show how flourishing social interaction research is and how interesting and important it is to take into account all these perspectives in order to expand our knowledge about the way in which human beings communicate in interaction with others. The conference gathered a wide range of scientific work providing an overview of the multifaceted phenomenon of social interaction at different levels but also on a continuum: From people's micro-level conduct in communication (in natural settings as well as in more experimental situations) to the mental mechanisms involved in communication underpinning cognitive abilities such as intentionality or mindreading. In a way, all the presentations invite us to expand our awareness on communication and social interaction by associating them as different branches of the same tree.

To conclude, this conference emphasised how important it is to build interdisciplinary collaborations in the field of communication and more broadly in social interaction studies. We hope that the discussions and debates that took place during these days also contribute to the formation of a common ground between the various disciplines represented in this review, while also promoting scientific collaborations between young researchers and experienced scholars faced with the challenge of analysing social interaction.

\section{Acknowledgments}

We would like to sincerely thank all the people who contributed to the success of 
the conference: The CRSI and its doctoral school, all attending participants and among them in particular all speakers and the organizing committee at the University of Neuchâtel.

\section{List of presenters at the CRSI-YR conference}

Hazbi AVDIJI (University of Lausanne) Adesignapproachtodiscursivecoordination

Geraldine BENGSCH (University of York) Interactional structures and communication sequences at the international authors front desk

Christina BRANDENBERGER \& Christoph HOTTIGER (University of Zurich) Making individual sensorial experiences available in interaction: A video and eye-tracking based study of knowledge construction in science centres

Elizaveta CHERNYSHOVA (University of Lyon 2)

Collaborative explicitation sequences in conversation: Some considerations on formulations, candidate understandings and shared knowledge

Jean-Marc DEWAELE (Birkbeck College, University of London)

Multilingualism and Emotions at the intersection of sociolinguistic, pragmatic, psycholinguistic, psychological, forensic and educational research

Daniel DUKES (University of Neuchâtel) Affective social learning and emotion appreciation: Structuring socio-emotional interactions

Hua FAN (University of Neuchâtel) 'Magic mirror on mobile, who is the fairest of them all?': The Affordances of Digitally Retouched Selfies

SylviaGONZALEZ(UniversityofNeuchâtel) Impact of stress on referring processes during a storytelling task
Sara GRECO (Università della Svizzera italiana)

Studying argumentation as a means to manage conflict in social interaction: An interdisciplinary journey

MichèleGROSSEN(UniversityofLausanne) The contribution of dialogism to the study of interactions

Kenan HOCHULI (University of Zurich) Sharing the space we inhabit - rhythms of movement as a semiotic base for communication

Ekaterina KHONINEVA (European University at Saint Petersburg)

Communication with voice assistants: Strategies of resolving understandability problems

Miranda KRONFLI (Imperial College London)

"How did it feel?": Learner storytelling in simulation debrief

Beatrice LIGORIO (University of Bari) Designing blended university courses to support identity development

Gabriel MACEDO (Federal University of Pernambuco)

An argumentative pedagogical design: Argumentative interaction to promote cognitive development

Ricardo MACHADO \& Margarida CÉSAR (Universidade Nova de Lisboa)

Different languages, different mathematical reasoning?

Eric MAYOR (University of Neuchâtel) The role of gender roles in stress reactions to a mock job interview

Gaëlle MOLINARI (TECFA University of Geneva)

Emotions in Learning mediated by computer

Kevin MONIER (University Paul Valéry, Montpellier 3)

When others' constancy is mine 
Konstancja OPRYSZEK (University of Silesia)

Emotional Speech Block Syndrome (ESBS)

- a case study

Elodie PAZE (University of Neuchâtel)

Elaboration of a protocol for the assessment of narrative discourse in bilingual children

Alain PERUSSET (University of Neuchâtel) Comment peut-on formaliser une pratique? Une approche sémiotique

Cécile PETITJEAN (University of Neuchâtel)

The power of laughs: From face-to-face interactions to text-based conversations

Alicja PILCH (University of Silesia) Audio visual materials in pre-diagnosis of anxiety disorders

Katarzyna PIOTROWSKA (University of Fribourg)

Adversarial sequences between journalist and interviewee in radio political interviews

Rebecca SCHÄR (Università della Svizzera italiana)

Negotiation of the issue in discussions among small children and their parents

Baya SEBIANE (University Paul Valéry, Montpellier 3)

Comparative study of the effects of favorite music on the gestural memory in 'normal' aging

Azza Akram SHAFEI (University of Geneva)

Higher education in Egypt: Development, quality and privatization

Klara SKOGMYR MARIAN (University of Neuchâtel)

Sequential development and upgrade practices in au-pair - child directives: $A$ case study of a low-proficiency L2 French speaker
Sonia SZRAMEK-KARCZ (University of Silesia)

Intentional (non-native) bilingualism in social interaction

Antonia STEGER (University of Zurich) Lingering in Public. Patterns of social interactions on urban squares

Ottilie TILSTON (University of Neuchâtel)

Understanding Latino Collectivism:

Cross-cultural Variation in Social Interaction with weak ties and strangers in Argentina, Britain and Canada

Claudia VENTURA \& Margarida CÉSAR (Universidad Nova de Lisboa)

Studying social interactions in learning and in research: How method shapes results

Anita ZYTOWICZ (University of Casimir-Le-Grand) Perception of life in England and feelings that come with language brokering from the perspective of multilingual Polish teenagers

Klaus ZUBERBÜHLER (University of Neuchâtel) Intentional communication in nonhuman primates? 

